\title{
Theories of Vision
}

\author{
By Dr. F. W. Edridge-Green, C.B.E.
}

$\mathrm{T}^{\mathrm{n}}$ HOUGH the subject of theories of vision has been under discussion for many years, it has been suggested to me that a useful purpose would be served by a statement of the case for the visual purple theory in comparison with the duplicity theory. The visual purple view is that the cones are the sole percipient elements of the retina, being stimulated by the photochemical decomposition of the liquid surrounding them, the rods not being percipient elements but nerve elements regulating the supply of visual purple to the liquid surrounding the cones. Direct stimulation of the colourless transparent cones is against all photochemical laws, and these colourless transparent cones are surrounded by a highly absorbent photochemical substance, the visual purple, which when decomposed must produce an effect.

Visual purple was found in liquid form in the retina surrounding, but not in the cones, by Devereux Marshall and me with monkeys' retinæ examined immediately after removal of the eyes. This experiment is supported by the fact that Kuhne found visual purple in a fluid form in the retina and poured it out on a plate.

There are numerous physiological facts, as, for example, the control of vision by the nervous system, the removal of waste products and the resensitisation of the cones, which are inexplicable on a direct stimulation hypothesis ${ }^{1}$. If the rods and cones were distinct percipient elements there should be a distinct qualitative difference between the portion of the retina containing only cones and that containing rods, but von Tschermak, Hering, Hess, Garten and others have found only gradual quantitative differences in the sight between the foveal and the extra foveal area. The Purkinje phenomenon, the alteration of optical white equations by the state of dark adaptation, the colourless interval for spectral lights of ircreasing intensity, the different phases of the after-image, all exist, not only in the extra foveal, but also, only gradually diminished, in the foveal region. In certain conditions the colour of the positive after-image of any colour or white is purple ${ }^{2}$.

Helmholtz pointed out that the evidence only proved that the cones were percipient elements and that there was no evidence that the rods were percipient elements. At a later date this view was reaffirmed by Nagel.

The eye resembles a photographic apparatus, and actual photographs have been taken on the retina and fixed by Kuhne. It will be noted that on the duplicity theory there is a very unsatisfactory correspondence with the film of a photographic apparatus. Photographers know that the sensitiveness of this film must be under control and a different sensitiveness is required for different intensities of light, but on the duplicity hypothesis there is only a mechanical arrangement. Bauer has shown that the visual purple is regenerated more rapidly under the stimulus of light than it is in absolute darkness, so that it must have a function in the daytime. If, however, we suppose that the function of the rods is to regulate the sensitiveness of the photographic film, that is to say, they are the nerve elements which liberate the visual purple into the liquid surrounding the cones, making this film more or less sensitive in accordance with the requirements of vision, we have an explanation of every known fact of vision.

The decomposition of the visual purple surrounding the cones causes stimulation of the cones and a visual impulse is set up. It is difficult to see how from the stimulation within the cone hypothesis the waste products can escape and a cone return to a condition in which it is able to receive further stimulation. It is probable that even in the early forms of vision, stimulation of the nerve ends took place not through the direct stimulation of the nerve end by light but indirectly through some light-absorbing substance which when acted upon by light gave off products which stimulated the nerve end. This appears to be the case even with the vision of insects.

The following are given as facts supporting the duplicity theory:-(1) that certain animals have only cones and others have only rods; (2) that the periphery of the retina is colour-blind; (3) that the eye is totally colour-blind in dark adaptation; (4) that the Purkinje phenomenon and the recurrent image are not found with the fovea; (5) that Portergraphs consist of two branches, one the branch corresponding to light above 0.25 metre-candle, corresponding to the cones, and the branch for light below $0 \cdot 25$, corresponding to the rods. With regard to these, I make the following comments :-

Though I have examined numerous collections, I have never been able to find any animal with only rods or only cones, neither have I met anyone who has seen such a retina. The tortoise is the most quoted; it is stated to have only cones. The rods and cones in the retina of the tortoise are as clearly defined and distinct as in the human retina. The periphery of the retina is not colour-blind when colours of sufficient intensity are used. This can readily be tested with a doctor's red lamp, which, it will be found, can be seen as red to the extreme periphery. In dark adaptation the eye is not totally colour-blind. Further, there is no scotoma or blind area corresponding to the rod-free portion of the macula which is equal to a visual angle of about three degrees. The Purkinje phenomenon and recurrent image are found with the fovea.

Frank Allen states that in his experiments all branches of the Porter-graphs were obtained on the same central area of the retina where rods are fewest in number or are altogether absent, the flickering at the point of fixation being specially noted. The presence of $B$ branches in the graphs obtained under these conditions is therefore quite adverse to the duplicity theory. The larger number of branches of the Porter-graphs and their identification with enhancing and depressing reflex actions seem to remove the graphs completely from their support of the duplicity theory. 
Secondly, facts which are explained in some measure by the duplicity theory can be explained in a far better manner by the visual purple theory. For example, the Purkinje phenomenon is found with other photochemical substances so that it is only a photochemical phenomenon. The visual purple gives a curve which is very similar to that of many other photochemical substances. We know that with photochemical substances the chemical effect is not proportional to the intensity of the light: a different curve is obtained with weak light from that which is formed with light of greater intensity. It is reasonable, therefore, to suppose that the visual purple which is formed by the pigment cells under the influence of a bright light would be somewhat different in character from that which is formed in darkness. Again, from the chemical analogy just given, even if the visual purple were of the same character we should not expect similar curves with different intensities of light. It is probable that both factors are in operation.

On the duplicity theory, the decomposition of the visual purple is supposed to stimulate the rods directly, but what other organ in the body produces a secretion which stimulates itself ?

The fact that a small star is seen more clearly on the para-foveal region than with the foveal region has been given as a strong fact supporting the duplicity theory, but the yellow pigment of the yellow spot has been entirely overlooked. This absorbs light, particularly blue, and would account for the phenomenon. Pertz has shown that the dark adapted fovea is more sensitive to red light than the periphery, and yet red light bleaches the visual purple. Again, the most sensitive portion of the dark adapted retina forms an oval with its long axis horizontal, the oval being ten degrees in its vertical axis and fifteen to twentydegrees initshorizontalaxis. This is just at the edge of the yellow spot where there are few rods. If this were a rod phenomenon, the light should appear much brighter farther out where the rods are much more numerous. The phenomenon is also found with the light adapted eye. If two discs of white paper each of a diameter of a sixteenth of an inch be pasted on black cardboard an inch apart and viewed from a distance of twelve to eighteen inches it will be found that the one that is directly looked at (with one eye) appears much darker and yellower than the other.

Congenital night blindness has been supposed to be due to absence of rods, but a better explanation is that the cones are not so sensitive as normal to a feeble stimulation, especially as this condition can be produced by continuous exposure by day to a bright light, as, for example, that reflected from snow. Congenital day blindness has been supposed to be due to absence of the cones, but a better explanation is that the mechanism of light adaptation is defective, probably from defective development of the rod and cone apparatus. This is supported by the fact that many of these persons see red imperfectly, and the rods are not supposed to convey impulses leading to the perception of colour.

In light adaptation, more visual purple is used up, but more is formed. In dark adaptation, though less is formed, much less is used up and so accumulates in the peripheral regions. The sensitisation of the foveal region from the periphery appears to be directly under the control of the nervous system.

We now come to facts which are inexplicable by the duplicity theory : the presence of visual purple in liquid form in the retina and surrounding but not in the cones; the fact that vision is plainly under the control of the nervous system is not explained.

At one moment the fovea appears the least sensitive portion of the retina and at the next moment may be the most sensitive portion of the retina. Helmholtz, whilst recording the fact, confessed that he was quite unable to suggest an explanation. The following simple experiment illustrates this :-On opening an eye on awaking in the morning and looking at the ceiling the central portion is seen as an irregular, circular, rhomboidal or star-shaped black spot. On closing the eye again a bluish violet circle appears at the periphery or middle of the field of vision, contracts and then after breaking up into a star-shaped figure and becoming brighter disappears, to be followed by another contracting circle. If the eye be opened when the star figure has formed in the centre it will appear as a bright, rose-coloured star, much brighter than any other part of the field of vision. If, however, we wait until the star has broken up and disappeared before opening the eye, it will be found that only a black spot is seen in the centre. This is explained on the theory that when there is visual purple in the fovea this is the most sensitive portion of the retina; when there is none there it is blind. It also shows that the fovea is sensitised from the periphery.

There are numerous methods by which currents in the field of vision which are not due to the circulation can be seen. The currents carry the visual quality, colour and brightness of the region from whence they come into an after-image. They also tend to move an after-image towards the centre; if we have two similar after-images, one situated in the centre and the other a short distance from the centre, the one external to the centre may be carried into the centre and combine with the one already there. These currents are formed by the flow of sensitised liquid.

The following simple experiment shows the influence of the para-foveal regions on the foveal region of the retina. Let a piece of black cardboard eight inches square be taken and place this on a wallpaper with a coloured pattern. The light in the room should not be too bright. The black cardboard should be viewed with one eye at a distance of six feet, the eye being kept as immovable as possible. It will then be noticed that portions of the colours of the wallpaper will appear to detach themselves from the wallpaper and move with a slow spiral motion into the black area. This will go on until the whole black area has completely disappeared, the surface being covered with a mixture of colours similar to those on the wallpaper.

There are numerous variations of the experiment. If a piece of yellow paper be placed on a blue ground and viewed as above, it will be noticed that the blue will invade the yellow until the latter appears quite white and then gradually becomes blue.

The bending of the re-current image at the fovea discovered by Hess is not explained by the duplicity theory. The duplicity theory fails to explain the change in position in the field of vision of afterimages on movement of the eye.

The cones, therefore, are the sole percipient elements, and on being stimulated start impulses which are conveyed along the optic nerve to the brain and stimulate certain cells of the visual centre causing a sensation of light, and other cells causing a sensation of colour.

1"The Physiology, of Vision" (G. Bell and Sons, 1920). "Science

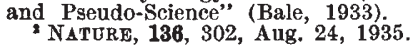

